\title{
Homotopic Approximate Solutions for the Perturbed CKdV Equation with Variable Coefficients
}

\author{
Dianchen Lu, Tingting Chen, and Baojian Hong \\ Center of Nonlinear Science Research, Jiangsu University, Zhenjiang, Jiangsu 212013, China \\ Correspondence should be addressed to Dianchen Lu; dclu@ujs.edu.cn
}

Received 21 November 2013; Accepted 15 January 2014; Published 5 March 2014

Academic Editors: T. Fang and X. Jing

Copyright (C) 2014 Dianchen Lu et al. This is an open access article distributed under the Creative Commons Attribution License, which permits unrestricted use, distribution, and reproduction in any medium, provided the original work is properly cited.

\begin{abstract}
This work concerns how to find the double periodic form of approximate solutions of the perturbed combined KdV (CKdV) equation with variable coefficients by using the homotopic mapping method. The obtained solutions may degenerate into the approximate solutions of hyperbolic function form and the approximate solutions of trigonometric function form in the limit cases. Moreover, the first order approximate solutions and the second order approximate solutions of the variable coefficients $\mathrm{CKdV}$ equation in perturbation $\varepsilon u^{n}$ are also induced.
\end{abstract}

\section{Introduction}

To solve the nonlinear partial differential equation (NPDE) has been an attractive research topic for mathematicians and physicists. Nonlinear evolution equations with variable coefficients can describe the physical phenomenon more accurately, and it is of great significance to study how to find the solutions of nonlinear evolution equations with variable coefficients.

In recent years, many researchers have developed various approaches for attaining the exact solutions and approximate of NPDE, such as the inverse scattering method [1], homogeneous balance method [2], elliptic function method [3], and perturbation method [4]. A recently reported analytic approximate method, the homotopic mapping method proposed in [5], has been applied to solve many nonlinear problems in engineering and technology effectively, like the nonlinear vibration of [6], boundary layer flow of [7], and so on [8-12]. However, the above works only studied the soliton approximate solutions of equations with constant coefficients. In this work, we applied the homotopic mapping method to the variable coefficients perturbed CKdV equation and obtained the approximate solution of the Jacobi elliptic function form.

\section{Model and Homotopy Mapping}

In this work, we focus on the perturbed CKdV equation with variable coefficients

$$
u_{t}+a(t) u u_{x}+b(t) u^{2} u_{x}+c(t) u_{3 x}=f\left(x, t, u, u_{x}, u_{t}\right),
$$

where $a(t), b(t), c(t)$ is any function about $t, f$ is a disturbance term, and $f$ is the sufficiently smooth function. This equation is widely used in the field of plasma physics [13], fluid mechanics [14], and quantum field theory [15]. It is fascinating to observe that when $a(t), b(t), c(t)$ is constant, $f=0$, (1) becomes the well-known combined KdV equation, the equation in plasma physics which describes the acoustic wave propagation of a small-amplitude ion without Landau decay. It can be used as a model equation in fluid mechanics; related research can be referred to in $[16,17]$.

When $f=R(t)$, (1) becomes the forced combined KdV equation, and the exact solutions of various forms are given in $[18,19]$, such as solitary wave solutions, trigonometric function solutions, and Jacobian elliptic function solutions. When $b(t)=0$, [20] studied the elliptic function solution of composite form. 
Next, we study the approximate solution of (1). In order to simplify (1), set

$$
\begin{gathered}
x^{\prime}=\sqrt{\frac{c_{3} a(t)}{c_{1} c(t)}} x, \quad t^{\prime}=\int_{0}^{t} \sqrt{\frac{c_{3} a^{3}(\tau)}{c_{1}^{3} c(\tau)}} d \tau, \\
t_{t}^{\prime}=\sqrt{\frac{c_{3} a^{3}(t)}{c_{1}^{3} c(t)}}
\end{gathered}
$$

where $c_{1}, c_{3}$ are any constants.

By setting $c_{2}(t)=b(t) c_{1} / a(t),(1)$ can be expressed as

$$
u_{t^{\prime}}+c_{1} u u_{x^{\prime}}+c_{2}(t) u^{2} u_{x^{\prime}}+c_{3} u_{3 x^{\prime}}=f^{*}\left(x^{\prime}, t^{\prime}, u, u_{x^{\prime}}, u_{t^{\prime}}\right)
$$

where

$$
\begin{gathered}
f^{*}\left(x^{\prime}, t^{\prime}, u, u_{x^{\prime}}, u_{t^{\prime}}\right) \\
=\left(f \left[\sqrt{\frac{c_{1} c(t)}{c_{3} a(t)}} x^{\prime}, t\left(t^{\prime}\right), u, \sqrt{\frac{c_{3} a(t)}{c_{1} c(t)}} u_{x^{\prime}},\right.\right. \\
\left.\left.\frac{b(t)}{c_{2}} \sqrt{\frac{c_{3} a^{3}(t)}{c_{1}^{3} c(t)}} u_{t^{\prime}}\right]\right) \times\left(t_{t}^{\prime}\right)^{-1} .
\end{gathered}
$$

For the sake of convenience, let $x=x^{\prime}, t=$ $t^{\prime}, f\left(x, t, u, u_{x}, u_{t}\right)=f^{*}\left(x^{\prime}, t^{\prime}, u, u_{x^{\prime}}, u_{t^{\prime}}\right)$, and (3) can be written as

$$
u_{t}+c_{1} u u_{x}+c_{3} u_{3 x}=-c_{2}(t) u^{2} u_{x}+f\left(x, t, u, u_{x}, u_{t}\right)
$$

the study on the solution of (1) is translated into the solution of (5).

In order to get the solution of (5), we lead in homotopy mapping.

2.1. Introduction of Homotopy Mapping. Assume we are given nonlinear equation $A(u)-f(r)=0, r \in \Omega$ and boundary condition $B(u, \partial u / \partial n)=0, r \in \Gamma$, where $A$ is the general differential operator, $B$ is the boundary operator, $f(r)$ is the known analytic function, and $\Gamma$ is the boundary of the region $\Omega$. Generally speaking, the operator $A$ can be decomposed into linear part $L$ and nonlinear part $N$. So equation $A(u)-$ $f(r)=0$ can be written as $L(u)+N(u)-f(r)=0$. $R$,

Now we set up homotopy mapping: $H(u, p): \Omega \times[0,1] \rightarrow$

$$
H(u, p)=L(u)-L(v)+p(L(v)+N(u)-f(r)),
$$

where $p$ is parameter, $v$ is auxiliary function, and $L(v)+$ $N(v)=0$.

By (6), we obtain

$$
\begin{gathered}
H(u, 0)=L(u)-L(v), \\
H(u, 1)=A(u)-f(r)=0 .
\end{gathered}
$$

As can be seen from 0 to 1 of $p$ is the process of $L(u)-L(v)$ to $A(u)-f(r)$ of $H(u, p)$; this is the homotopy deformation. Set

$$
\widetilde{u}(x, t, p)=\sum_{i=0}^{\infty} u_{i}(x, t) p^{i}=u_{0}+p u_{1}+p^{2} u_{2}+\cdots
$$

is the solution of $H(u, p)=0$. So when $p=0, \widetilde{u}(x, t, 0)=$ $u_{0}(x, t)$ is the solution of $L(u)-L(v)=0$; when $p \rightarrow 1$, the approximate solution of $A(u)-f(r)=0$ is $u(x, t)=u_{0}+u_{1}+$ $u_{2}+\cdots$.

2.2. Approximate Solution of the Jacobi Elliptic Function Form. Aiming at (5), we set up homotopy mapping $H(u, p): R \times$ $I \rightarrow R$,

$$
\begin{aligned}
H(u, p)= & L(u)-L(v) \\
& +p\left(L(v)+c_{1} u u_{x}\right. \\
& \left.\quad+c_{2}(t) u^{2} u_{x}-f\left(x, t, u, u_{x}, u_{t}\right)\right),
\end{aligned}
$$

where $R=(-\infty,+\infty), I=[0,1], v$ is the auxiliary function; linear operator $L$ is expressed as $L(u)=u_{t}+c_{3} u_{3 x}$.

By using the generalized ellipse method [21], we can get that the typical $\mathrm{KdV}$ equation corresponding to (5)

$$
v_{t}+c_{1} v v_{x}+c_{3} v_{3 x}=0
$$

has the following elliptic function solution:

$$
\begin{aligned}
v_{1}(x, t)= & c_{0}-\frac{6 k^{2} c_{3} m^{2} s n(\xi, m)+12 k^{2} c_{3}\left(m^{2}-1\right) c n(\xi, m)}{c_{1}(s n(\xi, m)+c n(\xi, m)+d n(\xi, m))} \\
& +\frac{3 k^{2} c_{3} m^{4} s n^{2}(\xi, m)-12 k^{2} c_{3} c n^{2}(\xi, m)}{c_{1}(s n(\xi, m)+c n(\xi, m)+d n(\xi, m))^{2}} .
\end{aligned}
$$

When $m \rightarrow 1, v_{1}(x, t)$ degenerates to the following solitary wave solution:

$$
\begin{aligned}
v_{1.1}(x, t)= & c_{0}+\frac{-6 k^{2} c_{3} \tanh \xi}{c_{1}(\tanh \xi+2 \sec h \xi)} \\
& +\frac{3 k^{2} c_{3} \tanh ^{2} \xi-12 k^{2} c_{3} \operatorname{sech}^{2} \xi}{c_{1}(\tanh \xi+2 \sec h \xi)^{2}} .
\end{aligned}
$$

When $m \rightarrow 0, v_{1}(x, t)$ degenerates to the trigonometric function solution

$$
\begin{aligned}
v_{1.2}(x, t)= & c_{0}+\frac{12 k^{2} c_{3} \cos \xi}{c_{1}(\sin \xi+\cos \xi+1)} \\
& -\frac{12 k^{2} c_{3} \cos ^{2} \xi}{c_{1}(\sin \xi+\cos \xi+1)^{2}},
\end{aligned}
$$

where $\xi=k x+\left[-c_{0} k c_{1}+k^{3} c_{3}\left(4 m^{2}-5\right)\right] t+\xi_{0}, k, c_{0}, \xi_{0}$ is any constant, $m$ is the module, and $0 \leq m \leq 1$.

One can easily prove that $H(u, 1)=0$ and (5) is the same, so the solution $u(x, t)$ of $(5)$ is the solution of $H(u, p)=0$ when under the condition $p \rightarrow 1$. 
Let

$$
\widetilde{u}(x, t, p)=\sum_{i=0}^{\infty} u_{i}(x, t) p^{i}=u_{0}+p u_{1}+p^{2} u_{2}+\cdots
$$

be the solution of $H(u, p)=0$; by [22] we can know this series is uniformly convergent in the $p \in[0,1]$. Thus, it yields that

$$
u=\sum_{i=0}^{\infty} u_{i}(x, t)=u_{0}+u_{1}+u_{2}+\cdots
$$

\section{Approximate Solution}

In order to obtain the approximate solution of (5), we substitute (14) into equation $H(u, p)=0$. By taking the auxiliary function $v=v_{1}(x, t)$ and comparing the coefficients of the same power of $p$, one can obtain that

$$
\begin{gathered}
p^{0}: L\left(u_{0}\right)=L(v)=L\left(v_{1}\right) \\
p^{1}: L\left(u_{1}\right)=- \\
+f\left(v_{1}\right)-c_{1} u_{0} u_{0 x}-c_{2}(t) u_{0}^{2} u_{0 x} \\
+f\left(x, t, u_{0}, u_{0 x}, u_{0 t}\right) \\
=-c_{2}(t) u_{0}^{2} u_{0 x}+f\left(x, t, u_{0}, u_{0 x}, u_{0 t}\right) \\
p^{2}: L\left(u_{2}\right)=-c_{1} u_{0} u_{1 x}-c_{1} u_{1} u_{0 x}-c_{2}(t) u_{0}^{2} u_{1 x}+F\left(u_{0}, u_{1}\right)
\end{gathered}
$$

where $F\left(u_{0}, u_{1}\right)=\left.(\partial / \partial p) f\left(x, t, \widetilde{u}, \tilde{u}_{x}, \tilde{u}_{t}\right)\right|_{p=0}$.

From (16) we have

$$
u_{0}(x, t)=v_{1}(x, t)
$$

By using the Fourier transform, one can obtain the solution of (17) with the initial condition $\left.u_{1}\right|_{t=0}=0$ as follows:

$$
\begin{aligned}
& u_{1}(x, t) \\
& =\frac{1}{2 \pi} \int_{0}^{t} \int_{-\infty}^{+\infty}\left[-c_{2}(\tau) u_{0}^{2} u_{0 \xi}+f\left(\xi, \tau, u_{0},\left(u_{0}\right)_{\xi},\left(u_{0}\right)_{\tau}\right)\right] \\
& \quad \times \int_{-\infty}^{+\infty} \cos \left[-\lambda^{3} c_{3}(t-\tau)+\lambda(x-\xi)\right] d x d \xi d \tau
\end{aligned}
$$

Similarly, one also finds the solution of (18) with the initial condition $\left.u_{2}\right|_{t=0}=0$

$$
\begin{aligned}
& u_{2}(x, t) \\
& =\frac{1}{2 \pi} \int_{0}^{t} \int_{-\infty}^{+\infty}\left(-c_{1} u_{0} u_{1 x}-c_{1} u_{1} u_{0 x}-c_{2}(t) u_{0}^{2} u_{1 x}+F\left(u_{0}, u_{1}\right)\right) \\
& \quad \times \int_{-\infty}^{+\infty} \cos \left[-\lambda^{3} c_{3}(t-\tau)+\lambda(x-\xi)\right] d x d \xi d \tau
\end{aligned}
$$

From (11), (12), (13), (20), and (21), the two degree approximate solutions of (5) can be obtained

$$
\begin{aligned}
& u_{2}^{*}(x, t) \\
& =c_{0}-\frac{6 k^{2} c_{3} m^{2} s n(\xi, m)+12 k^{2} c_{3}\left(m^{2}-1\right) c n(\xi, m)}{c_{1}(s n(\xi, m)+c n(\xi, m)+d n(\xi, m))} \\
& +\frac{3 k^{2} c_{3} m^{4} s^{2}(\xi, m)-12 k^{2} c_{3} c n^{2}(\xi, m)}{c_{1}(s n(\xi, m)+c n(\xi, m)+d n(\xi, m))^{2}} \\
& +\frac{1}{2 \pi} \int_{0}^{t} \int_{-\infty}^{+\infty}\left(-c_{2}(\tau) u_{0}^{2} u_{0 \xi}+f\left(\xi, \tau, u_{0},\left(u_{0}\right)_{\xi},\left(u_{0}\right)_{\tau}\right)\right. \\
& \quad-c_{1} u_{0} u_{1 x}-c_{1} u_{1} u_{0 x} \\
& \quad \int_{-\infty}^{+\infty} \cos \left[-\lambda_{2}^{3} c_{3}(t) u_{0}^{2} u_{1 x}+F\left(u_{0}, u_{1}\right)\right)
\end{aligned}
$$

where $\xi=k x+\left[-c_{0} k c_{1}+k^{3} c_{3}\left(4 m^{2}-5\right)\right] t+\xi_{0}, k, c_{0}, \xi_{0}$ is any constant, $m$ is the module, and $0 \leq m \leq 1, F\left(u_{0}, u_{1}\right)=$ $\left.(\partial / \partial p) f\left(x, t, \tilde{u}, \tilde{u}_{x}, \tilde{u}_{t}\right)\right|_{p=0}$,

$$
\begin{aligned}
& u_{0}= c_{0}-\frac{6 k^{2} c_{3} m^{2} s n(\xi, m)+12 k^{2} c_{3}\left(m^{2}-1\right) c n(\xi, m)}{c_{1}(s n(\xi, m)+c n(\xi, m)+d n(\xi, m))} \\
&+ \frac{3 k^{2} c_{3} m^{4} s n^{2}(\xi, m)-12 k^{2} c_{3} c n^{2}(\xi, m)}{c_{1}(s n(\xi, m)+c n(\xi, m)+d n(\xi, m))^{2}}, \\
& u_{1}=\frac{1}{2 \pi} \int_{0}^{t} \int_{-\infty}^{+\infty}\left[-c_{2}(\tau) u_{0}^{2} u_{0 \xi}+f\left(\xi, \tau, u_{0},\left(u_{0}\right)_{\xi},\left(u_{0}\right)_{\tau}\right)\right] \\
& \quad \times \int_{-\infty}^{+\infty} \cos \left[-\lambda^{3} c_{3}(t-\tau)+\lambda(x-\xi)\right] d x d \xi d \tau .
\end{aligned}
$$

When $m \rightarrow 1$ and $m \rightarrow 0, u_{2}^{*}(x, t)$ degenerates to the following approximate solutions:

$$
\begin{aligned}
& u_{2.1}^{*}(x, t) \\
& =c_{0}-\frac{6 k^{2} c_{3} \tanh \xi}{c_{1}(\tanh \xi+2 \sec h \xi)} \\
& +\frac{3 k^{2} c_{3} \tanh ^{2} \xi-12 k^{2} c_{3} \sec h^{2} \xi}{c_{1}(\tanh \xi+2 \operatorname{sech} \xi)^{2}} \\
& +\frac{1}{2 \pi} \int_{0}^{t} \int_{-\infty}^{+\infty}\left(-c_{2}(\tau) u_{0}^{2} u_{0 \xi}\right. \\
& +f\left(\xi, \tau, u_{0},\left(u_{0}\right)_{\xi},\left(u_{0}\right)_{\tau}\right) \\
& -c_{1} u_{0} u_{1 x}-c_{1} u_{1} u_{0 x}
\end{aligned}
$$




$$
\begin{gathered}
\left.-c_{2}(t) u_{0}^{2} u_{1 x}+F\left(u_{0}, u_{1}\right)\right) \\
\times \int_{-\infty}^{+\infty} \cos \left[-\lambda^{3} c_{3}(t-\tau)+\lambda(x-\xi)\right] d x d \xi d \tau, \\
u_{2.2}^{*}(x, t) \\
=c_{0}+\frac{-6 k^{2} c_{3} \sin \xi+12 k^{2} c_{3} \cos \xi}{c_{1}(\sin \xi+\cos \xi+1)}-\frac{12 k^{2} c_{3} \cos ^{2} \xi}{c_{1}(\sin \xi+\cos \xi+1)^{2}} \\
+\frac{1}{2 \pi} \int_{0}^{t} \int_{-\infty}^{+\infty}\left(-c_{2}(\tau) u_{0}^{2} u_{0 \xi}\right. \\
+f\left(\xi, \tau, u_{0},\left(u_{0}\right)_{\xi},\left(u_{0}\right)_{\tau}\right) \\
-c_{1} u_{0} u_{1 x}-c_{1} u_{1} u_{0 x} \\
\left.-c_{2}(t) u_{0}^{2} u_{1 x}+F\left(u_{0}, u_{1}\right)\right) \\
\times \int_{-\infty}^{+\infty} \cos \left[-\lambda^{3} c_{3}(t-\tau)+\lambda(x-\xi)\right] d x d \xi d \tau .
\end{gathered}
$$

By comparing the higher power coefficients of $p$, more higher power approximate solutions can also be obtained, and hence the approximate solution of (1) is found.

\section{Example}

If $f=\varepsilon u^{n}$ is the disturbance term of (5), where $0<\varepsilon \ll 1$, (5) becomes

$$
u_{t}+c_{1} u u_{x}+c_{2}(t) u^{2} u_{x}+c_{3} u_{3 x}=\varepsilon u^{n} .
$$

By using the above method in Section 3, one can find each order approximate solution of elliptic function form for (25) as follows:

$$
\begin{aligned}
u_{0}^{*}( & x, t) \\
= & c_{0}-\frac{6 k^{2} c_{3} m^{2} \operatorname{sn}(\xi, m)+12 k^{2} c_{3}\left(m^{2}-1\right) c n(\xi, m)}{c_{1}(s n(\xi, m)+c n(\xi, m)+d n(\xi, m))} \\
& +\frac{3 k^{2} c_{3} m^{4} s n^{2}(\xi, m)-12 k^{2} c_{3} c n^{2}(\xi, m)}{c_{1}(s n(\xi, m)+c n(\xi, m)+d n(\xi, m))^{2}}, \\
u_{1}^{*}(x, t) & \\
= & c_{0}-\frac{6 k^{2} c_{3} m^{2} \operatorname{sn}(\xi, m)+12 k^{2} c_{3}\left(m^{2}-1\right) c n(\xi, m)}{c_{1}(s n(\xi, m)+c n(\xi, m)+d n(\xi, m))} \\
& +\frac{3 k^{2} c_{3} m^{4} s n^{2}(\xi, m)-12 k^{2} c_{3} c n^{2}(\xi, m)}{c_{1}(s n(\xi, m)+c n(\xi, m)+d n(\xi, m))^{2}} \\
& +\frac{1}{2 \pi} \int_{0}^{t} \int_{-\infty}^{+\infty}\left(-c_{2}(\tau) u_{0}^{2} u_{0 \xi}+\varepsilon u_{0}^{n}\right) \\
& \quad \times \int_{-\infty}^{+\infty} \cos \left[-\lambda^{3} c_{3}(t-\tau)+\lambda(x-\xi)\right] d x d \xi d \tau,
\end{aligned}
$$

$$
\begin{aligned}
& u_{2}^{*}(x, t) \\
& =c_{0}-\frac{6 k^{2} c_{3} m^{2} s n(\xi, m)+12 k^{2} c_{3}\left(m^{2}-1\right) c n(\xi, m)}{c_{1}(s n(\xi, m)+c n(\xi, m)+d n(\xi, m))} \\
& +\frac{3 k^{2} c_{3} m^{4} s n^{2}(\xi, m)-12 k^{2} c_{3} c n^{2}(\xi, m)}{c_{1}(s n(\xi, m)+c n(\xi, m)+d n(\xi, m))^{2}} \\
& +\frac{1}{2 \pi} \int_{0}^{t} \int_{-\infty}^{+\infty}\left(-c_{2}(\tau) u_{0}^{2} u_{0 \xi}+\varepsilon u_{0}^{n}-c_{1} u_{0} u_{1 x}\right. \\
& \quad \times \int_{-\infty}^{+\infty} \cos \left[-\lambda_{1}^{3} c_{3}(t-\tau)+\lambda(x-\xi)\right] d x d \xi d \tau
\end{aligned}
$$

where $\xi=k x+\left[-c_{0} k c_{1}+k^{3} c_{3}\left(4 m^{2}-5\right)\right] t+\xi_{0}, k, c_{0}, \xi_{0}$ is any constant, $m$ is the module, and $0 \leq m \leq 1, u_{0}=u_{0}^{*}(x, t)$,

$$
\begin{aligned}
u_{1}=\frac{1}{2 \pi} \int_{0}^{t} \int_{-\infty}^{+\infty}\left[-c_{2}(\tau) u_{0}^{2} u_{0 \xi}+\varepsilon u_{0}^{n}\right] \\
\quad \times \int_{-\infty}^{+\infty} \cos \left[-\lambda^{3} c_{3}(t-\tau)+\lambda(x-\xi)\right] d x d \xi d \tau .
\end{aligned}
$$

\section{Conclusion}

This work studies the perturbed CKdV equation with variable coefficients by using the homotopic mapping method, and two degree approximate solution of the Jacobi elliptic function form are obtained, which can degenerate to solitary wave approximate solution and trigonometric function approximate solution in the limit cases. Furthermore, the approximate solution of the perturbed CKdV is also obtained. Our results show that the homotopic mapping method is applicable to the variable soliton equations. How to apply this method to high degree and high dimension system remains to be further studied.

\section{Conflict of Interests}

The authors declare that there is no conflict of interests regarding the publication of this paper.

\section{Acknowledgments}

This work is supported by the National Natural Science Foundation of China (no. 61070231), the Outstanding Personal Program in Six Fields of Jiangsu Province, China (Grant no. 2009188), and the Graduate Student Innovation Project of Jiangsu Province (Grant no. CXLX13_673).

\section{References}

[1] M. J. Ablowitz and P. A. Clarkson, Solitons. Nonlinear Evolution Equations and Inverse Scattering, Cambridge University Press, New York, NY, USA, 1991. 
[2] E. Fan, "Two new applications of the homogeneous balance method," Physics Letters A, vol. 265, no. 5-6, pp. 353-358, 2000.

[3] W. Huang and Y. Liu, "Jacobi elliptic function solutions of the Ablowitz-Ladik discrete nonlinear Schrödinger system," Chaos, Solitons and Fractals, vol. 40, no. 2, pp. 786-792, 2009.

[4] H. Fu, L. Cao, and B. Han, "A homotopy perturbation method for well log constrained seismic waveform inversion," Chinese Journal of Geophysics, vol. 55, no. 9, pp. 2173-2179, 2004.

[5] L. Shi and X. Zhou, "Homotopic mapping solution of soliton for a class of disturbed Burgers equation," Acta Physica Sinica, vol. 59, no. 5, pp. 2915-2918, 2010.

[6] Q. Zhang, W. Wang, and X. He, "Homoclinic bifurcation of the strongly nonlinear oscillation system by the normal form method," Acta Physica Sinica, vol. 57, no. 9, pp. 5384-5389, 2008.

[7] R. Li and C. Li, "A link between chaos dynamics and the onset of turbulence in a transitional boundary layer," Acta Physica Sinica, vol. 51, no. 8, pp. 1748-1749, 2002.

[8] Y. Shi, X. Xu, Z. Wu et al., "Application of the homotopy analysis method to solving nonlinear evolution equations," Acta Physica Sinica, vol. 55, no. 4, pp. 1555-1560, 2006.

[9] J. Mo and J. Yao, "Homotopic mapping solution of soliton for perturbed KdV equation," Acta Physica Sinica, vol. 57, no. 12, pp. 7419-7422, 2008.

[10] L. Shi and J. Mo, "Soliton-like homotopic approximate analytic solution for a class of disturbed nonlinear evolution equation," Acta Physica Sinica, vol. 58, no. 12, pp. 8123-8126, 2009.

[11] Z. Zhou, S. Ma, J. Fang, and Q. Ren, "Multi-soliton solutions and fractal structures in a $(2+1)$-dimensional soliton system," Acta Physica Sinica, vol. 59, no. 11, pp. 7540-7545, 2010.

[12] Y. Shi and H. Yang, "Application of the homotopy analysis method to solving dissipative system," Acta Physica Sinica, vol. 59, no. 1, pp. 67-74, 2010.

[13] G. Yang, "Plasma physics of optical film deposition by reactive ion plating," Semiconductor Optoelectronics, 1996-01.

[14] X. Han and R. Qian, "A study on the basic theory of fluid mechanics," Spatial Structures, no. 3, pp. 9-12, 2008.

[15] Z. Zheng and K. Wu, "Topological quantum field theory and crossing number," Acta Mathematiea Scientia, vol. 20, no. 4, pp. 493-498, 2000.

[16] Y. Shi, K. Lü, and W. Duan, "Explicit and exact solutions of the combined KdV equation," Acta Physica Sinica, vol. 52, no. 2, pp. 267-270, 2003.

[17] J. Pan and L. Gong, "Jacobi elliptic function solutions to the coupled KdV-mKdV equation," Acta Physica Sinica, vol. 56, no. 10, pp. 5585-5590, 2007.

[18] D. Lu, B. Hong, and L. Tian, "Explicit and exact solutions to the variable coefficient combined KdV equation with forced term," Acta Physica Sinica, vol. 55, no. 11, pp. 5617-5622, 2006.

[19] T. Taogetusang and S. Sirendaoerji, "The auxiliary equation for constructing the exact solutions of the variable coefficient combined KdV equation with forcible term," Acta Physica Sinica, vol. 57, no. 3, pp. 1295-1300, 2008.

[20] R. Sabry, M. A. Zahran, and E. Fan, "A new generalized expansion method and its application in finding explicit exact solutions for a generalized variable coefficients KdV equation," Physics Letters A, vol. 326, no. 1-2, pp. 93-101, 2004.

[21] B. Hong, "New Jacobi elliptic functions solutions for the variable-coefficient $\mathrm{mKdV}$ equation," Applied Mathematics and Computation, vol. 215, no. 8, pp. 2908-2913, 2009.

[22] S. Liao, Beyond Perturbation: Introduction to the Homotopy Analysis Method, CRC Press, New York, NY, USA, 2004. 


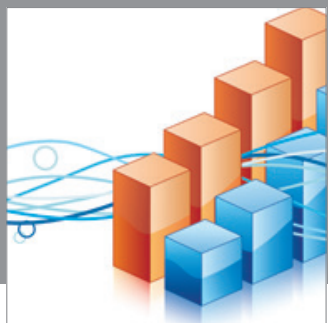

Advances in

Operations Research

mansans

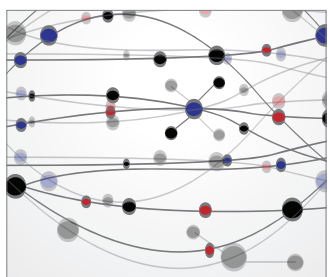

The Scientific World Journal
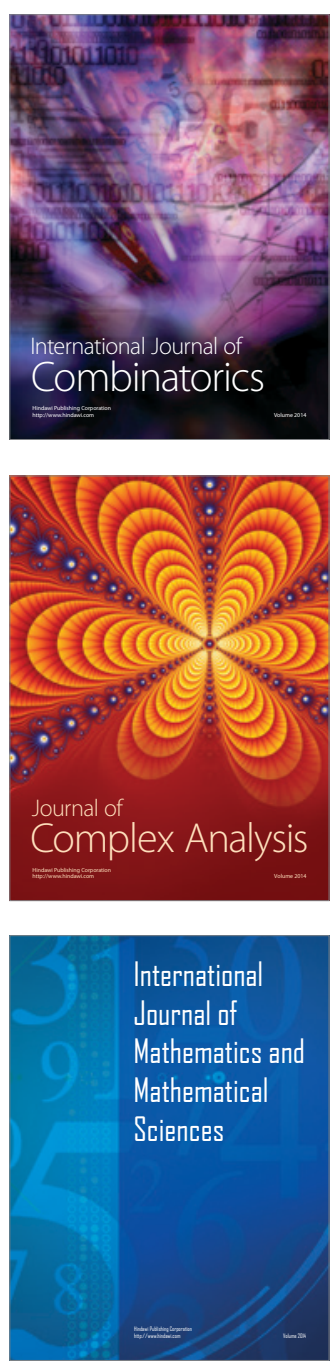
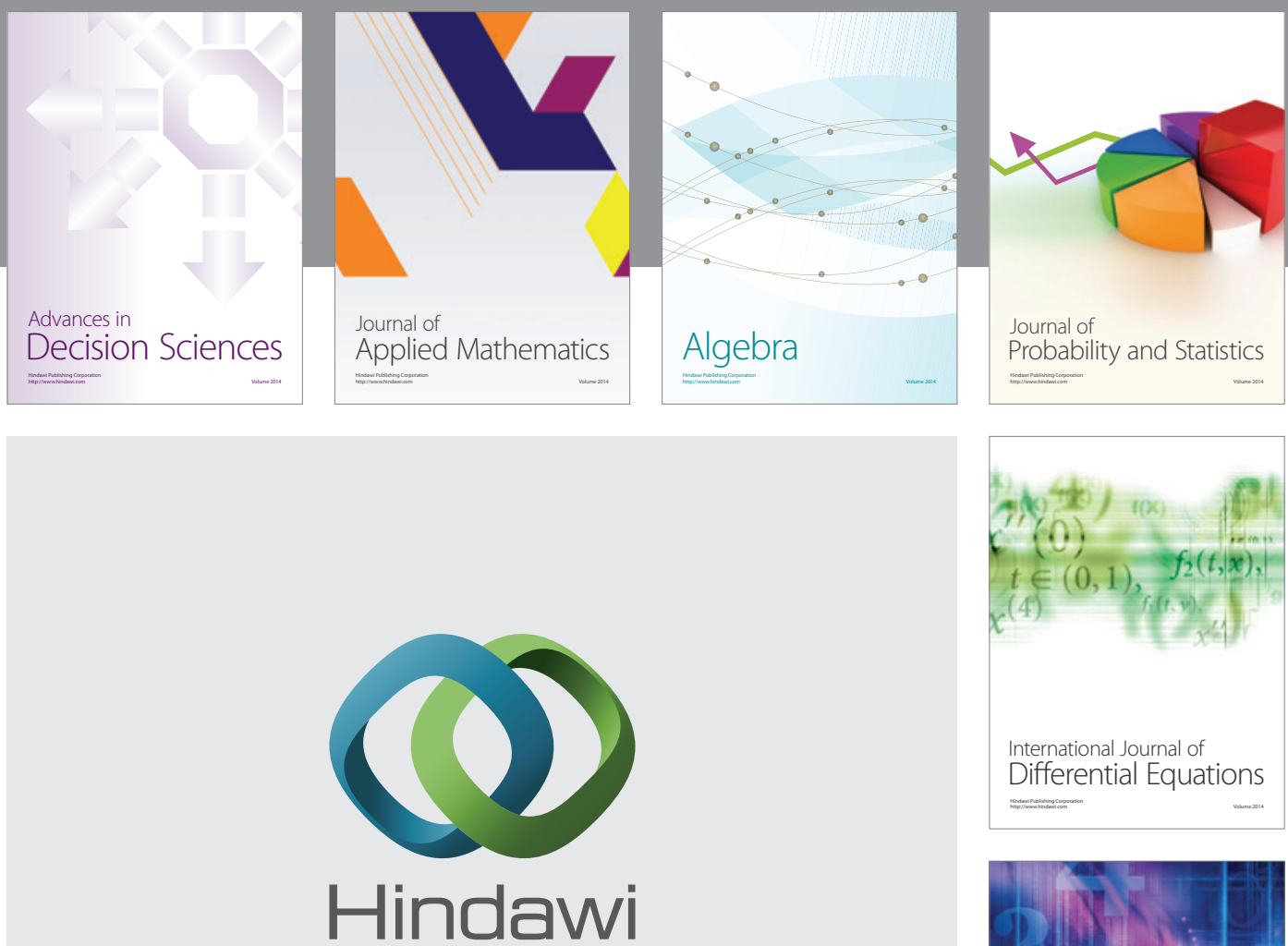

Submit your manuscripts at http://www.hindawi.com
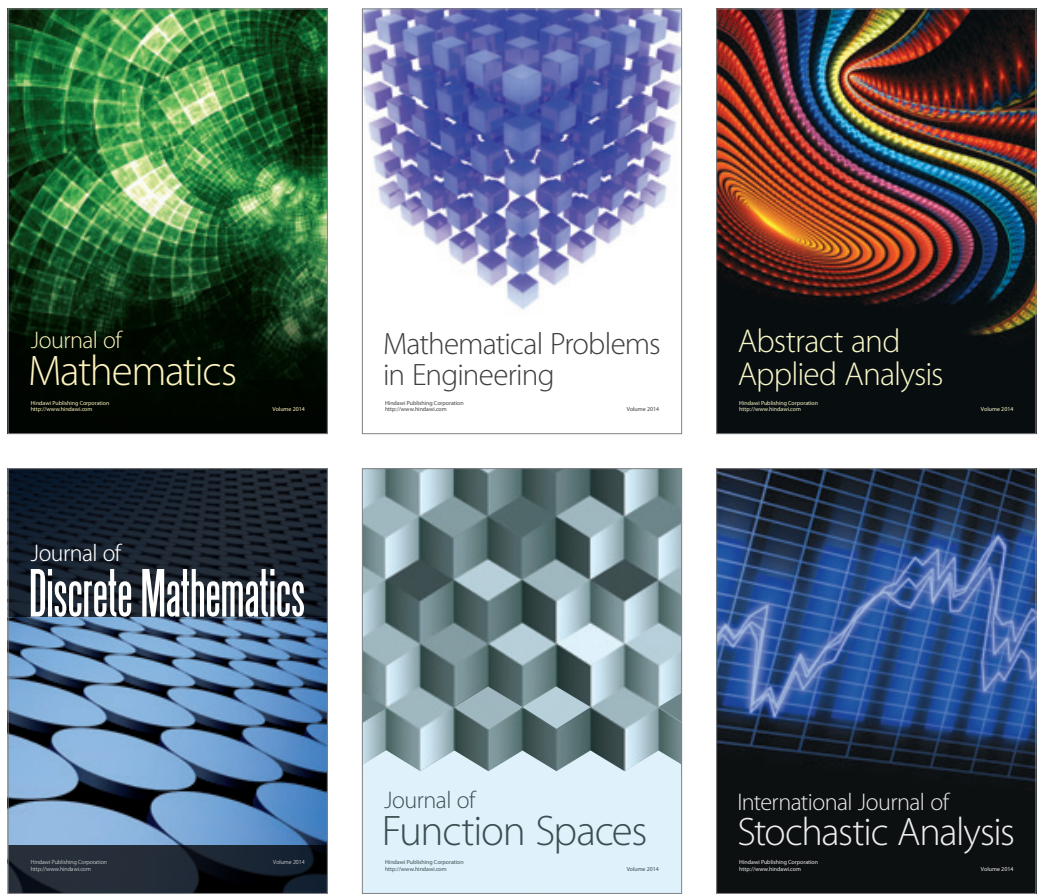

Journal of

Function Spaces

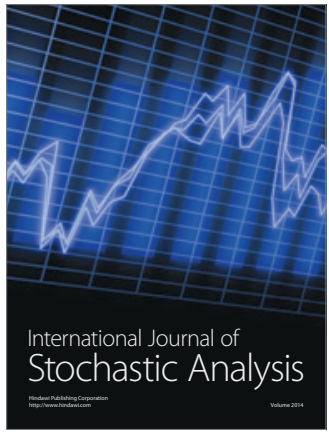

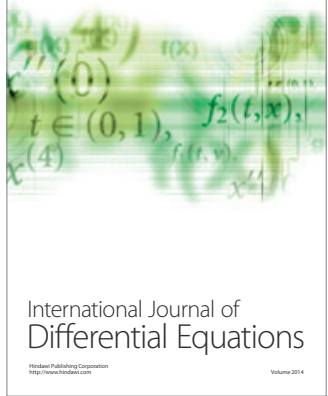
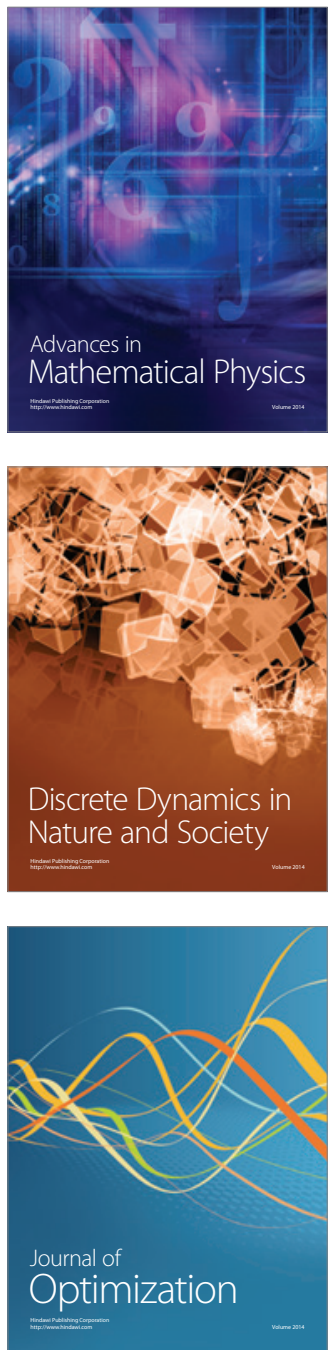